\title{
Maggiore ricerca per i farmaci generici
}

\author{
More research is needed for generic drugs
}

Può essere utile fare alcune considerazioni sui farmaci generici, detti anche equivalenti, rispetto ai farmaci con il nome di fantasia. Gli equivalenti hanno un grande successo in tutta Europa, ma sono ancora poco utilizzati e addirittura contestati in Italia. La scarsa utilizzazione (circa un terzo della media europea) dipende da molti fattori, tra cui il fatto che sono stati introdotti con ritardo perché in Italia è stato prolungato il brevetto per molti farmaci di marca. Un altro fattore importante dipende dalla diminuzione di prezzo che $i$ farmaci di marca hanno effettuato per competere con i farmaci equivalenti e mantenere le quote di mercato acquisite. Infine è importante ricordare che è più facile fare propaganda per un farmaco che ha un nome esclusivo rispetto ad un farmaco che ha un nome, appunto il nome generico, che è simile a quello di tanti altri.

La contestazione è generata dai dubbi che sono stati diffusi - evidentemente da chi ha interesse a farlo - circa l'efficacia di questi farmaci. I dubbi riguardano la qualità del principio attivo, il contenuto in eccipienti e la conseguente assorbibilità dei farmaci equivalenti. Si tratta di dubbi assolutamente ingiustificati alla luce dei dati scientifici disponibili: molti studi che hanno confrontato farmaci generici e farmaci di marca non hanno dimostrato significative differenze di efficacia.

Recentemente i prezzi dei farmaci generici sono stati diminuiti, spesso in modo consistente, per tener conto dei prezzi medi europei. Il Servizio Sanitario Nazionale rimborserà solo il generico con il prezzo più basso e chi vorrà acquistare il generico o il farmaco di marca che non avrà abbassato il prezzo dovrà pagare la differenza. La decisione presa dall'AIFA non fa una grinza perché di fronte ad un aumento di circa 1,5 miliardi di euro della spesa farmaceutica nel 2010 rispetto all'anno 2009 sembra logico adeguare i prezzi italiani a quelli europei quando a pagare è lo Stato ma con i soldi delle nostre tasse. L'unico appunto che si può fare è quello di non aver adeguatamente informato i cittadini con il dovuto anticipo e con le necessarie spiegazioni determinando così proteste e incomprensioni da parte del pubblico.

Mentre è giusto cercare di portare i consumi dei farmaci generici in Italia al livello degli altri Paesi europei, è importante evitare un pericolo che si sta delineando in modo chiaro. Quando un farmaco diventa generico spesso il suo mercato globale diminuisce e aumenta il mercato dei prodotti analoghi che mantengono ancora il brevetto. È il caso della ranitidina sostituita dagli omeprazoli, la simvastatina sostituita dall'atorvastatina o dalla rosuvastatina, e ancora degli ACE-inibitori sostituiti dai sartani. Man mano che passa il tempo è possibile che molti farmaci generici vengano abbandonati. Tuttavia questo non accade perché vengono superati da farmaci più attivi o meglio tollerati ma semplicemente perché non sono più oggetto di ricerca. Il Servizio Sanitario Nazionale deve essere interessato a sostenere questa ricerca per non perdere farmaci che hanno il vantaggio di essere non solo validi ma anche di minor costo. Si deve anche incentivare l'industria a continuare la ricerca per trovare nuove indicazioni, ma ciò richiede che l'industria abbia dei vantaggi, ad esempio ottenendo l'esclusività per le nuove indicazioni.

È tempo di occuparsi seriamente dei farmaci generici soprattutto perché in questo periodo non vi sono molti nuovi farmaci innovativi e si rischia di prendere per novità prodotti che in realtà sono meno efficaci dei generici.

Silvio Garattini

Direttore dell'Istituto di Ricerche Farmacologiche Mario Negri, Milano

E-mail: silvio.garattini@marionegri.it 